\title{
As práticas sexuais dos graduandos de enfermagem e a prevenção das doenças sexualmente transmissíveis
}

\section{Sexual practices of nursing undergraduates and prevention of sexually transmitted diseases \\ Las prácticas sexuales de los estudiantes de enfermería y la prevención de enfermedades de transmisión sexual}

\author{
Haisa Borges d'Amaral'; Laís de Andrade Rosa'; Raquel de Oliveira Wilken III, Thelma Spindola ${ }^{\mathrm{IV}}$; \\ Maria Regina Richerte Araujo PimentelV; Luiz Eduardo da Motta Ferreira ${ }^{\mathrm{Vl}}$
}

\begin{abstract}
RESUMO: Estudo descritivo, quantitativo realizado com informações do banco de dados da pesquisa Avaliando o conhecimento, as práticas e crenças dos estudantes universitários em relação às doenças sexualmente transmissíveis, com objetivo de identificar as práticas sexuais dos graduandos de enfermagem, a vulnerabilidade e as condutas adotadas para a prevenção dessas doenças. A pesquisa foi desenvolvida, em 2013/2014, em uma universidade pública, no município do Rio de Janeiro, com estudantes de graduação em enfermagem, que responderam a um questionário. Para compor esta investigação, extraiu-se uma amostra de 89 participantes. Os resultados indicam que os jovens não adotam o uso do preservativo de forma contínua, tornando-se vulneráveis às doenças sexualmente transmissíveis. Ações que contribuam na educação para a saúde dos jovens, com estímulo à adoção de práticas sexuais seguras, favorecem a prevenção da ocorrência de doenças sexualmente transmissíveis e devem ser intensificadas.
\end{abstract}

Palavras-Chave: Práticas sexuais; estudantes universitários; doenças sexualmente transmissíveis; prevenção.

\begin{abstract}
This quantitative, descriptive study drew on the database of the research Evaluating university students' knowledge, practices and beliefs in relation to sexually transmitted diseases in order to identify the sexual practices of nursing undergraduates, their vulnerability to STDs, and the attitudes and practices they deploy to prevent them. A questionnaire was applied to a sample of 89 undergraduate nursing students at a public university Rio de Janeiro City in 2013-2014. The results showed that these young people's use of condoms is not continuous, making them vulnerable to contracting sexually transmitted diseases. Actions that contribute to these young people's health education, including encouragement for their adopting safe sex practices, help to prevent the occurrence of sexually transmitted diseases and should be promoted.

Keywords: Sexual practices; university students; sexually transmitted diseases; prevention.

RESUMEN: Estudio descriptivo y cuantitativo realizado con material almacenado en los bancos de datos de la investigación Evaluando el conocimiento, las prácticas y creencias de los estudiantes universitarios en relación con las enfermedades de transmisión sexual, con el objetivo de identificar las prácticas sexuales de los estudiantes de enfermería, la vulnerabilidad y las prácticas adoptadas por los estudiantes para la prevención de esas enfermedades. Fue realizado, en 2013/2014, en una universidad pública, en la ciudad de Río de Janeiro junto a estudiantes de enfermería que respondieron a un cuestionario. Para componer esta investigación se extrajo uma muestra de 89 participantes. Los resultados evidencian que los jóvenes no adoptan el uso de condones de forma continua, lo que los vuelve vulnerables a las enfermedades de transmisión sexual. Acciones que contribuyan a la educación en la salud de los jóvenes, con incentivos a la adopción de prácticas sexuales seguras favorecen la prevención de la ocurrencia de enfermedades de transmisión sexual y deben ser estimuladas.

Palabras Clave: Prácticas sexuales; estudiantes universitarios; enfermedades de transmisión sexual; prevención.
\end{abstract}

\section{INTRODUÇÃO}

O objeto deste estudo são as práticas sexuais de jovens e a prevenção das doenças sexualmente transmissíveis ${ }^{\mathrm{VII}}$. A descoberta e iniciação sexual dos jovens, associada à prática do sexo de forma insegura, contribuem para que este grupo se torne a parcela da população mais vulnerável em contrair doenças, especialmente aquelas transmitidas pelo contato sexual. $\mathrm{O}$ desconhecimento dos meios de prevenção, as formas

IEnfermeira. Pós-Graduanda na modalidade de Residência em Enfermagem Obstétrica pela Universidade Federal Fluminense. Graduanda pela Faculdade de Enfermagem da Universidade do Estado do Rio de Janeiro. Brasil. E-mail: haisa.borges@gmail.com

IIEnfermeira. Pós-Graduanda na modalidade de Residência em Enfermagem Médico-Cirúrgica pela Universidade Federal do Estado do Rio de Janeiro. Graduada pela Faculdade de Enfermagem da Universidade do Estado do Rio de Janeiro. Brasil. E-mail: laisandraderosa@gmail.com.

IIIEnfermeira. Graduada pela Faculdade de Enfermagem da Universidade do Estado do Rio de Janeiro. Brasil. E-mail: raqueldeoliveirawilken@hotmail.com. IVEnfermeira. Doutora em Enfermagem. Professora Associada do Departamento de Fundamentos de Enfermagem da Faculdade de Enfermagem da Universidade do Estado do Rio de Janeiro. Brasil. E-mail: tspindola.uerj@gmail.com.

vEnfermeira. Doutoranda em Enfermagem. Professora Assistente do Departamento de Fundamentos de Enfermagem da Faculdade de Enfermagem da Universidade do Estado do Rio de Janeiro. Brasil.E-mail: mariaregina.pimentel85@gmail.com.

VIMédico. Mestre em Cardiologia. Professor Adjunto da Escola de Medicina e Cirurgia da Universidade Federal do Estado do Rio de Janeiro. Brasil.

E-mail: luizmotta@predialnet.com.br.

VIIParte integrante do relatório de pesquisa intitulado As práticas sexuais dos graduandos de enfermagem e a prevenção de doenças sexualmente transmissíveis, apresentado como pré-requisito para aprovação na Graduação em Enfermagem, da Universidade do Estado do Rio de Janeiro, em 2014. 
de contágio ou simplesmente adoção de comportamento de risco são situações a serem investigadas, uma vez que refletem na incidência das doenças sexualmente transmissíveis (DST) na população jovem. Entre as doenças que estão associadas à prática do sexo desprotegido, insere-se a Síndrome da Imunodeficiência Adquirida (AIDS), considerada um marco na epidemiologia mundial e responsável por mudanças significativas no âmbito da saúde, trazendo discussões acerca dos comportamentos sexuais, crenças, valores e mitos associados com a sexualidade ${ }^{1,2}$.

A sexualidade de um grupo social é determinada segundo valores, concepções e normas da sociedade a qual pertence, conforme o momento histórico que irá determinar o certo ou errado, apropriado ou inapropriado, de acordo com os padrões vigentes. Além das práticas sexuais, o desejo, o afeto, o prazer e as fantasias compõem a sexualidade ${ }^{3}$.

Dados do Ministério da Saúde indicam que, no período de 2000 a 2006, foram registrados 19.793 casos de aids em jovens com idades entre 13 e 24 anos no Brasil, representando $80 \%$ dos casos notificados. De acordo com o Boletim Epidemiológico DST/aids, existem, aproximadamente, 709.477 casos de aids identificados no Brasil desde 1980 até junho de 2013. No Rio de Janeiro, nos últimos cinco anos, houve um aumento no número de casos notificados de aids na população jovem, de 15 a 24 anos de idade, passando de $16,7 \%$ em 2008 para 19,3\% em 2013,5.

Alguns fatores podem ser apontados como responsáveis pelos índices de contaminação, entre eles a desinformação sobre o assunto e a falta de diálogo familiar para orientar seus jovens sobre sexualidade. Os pais têm dificuldade de dialogar sobre questões de sexualidade com os filhos, não só por constrangimento, mas também por medo que o diálogo franco e aberto possa indicar aos adolescentes que já estão prontos para iniciar a vida sexual. Autores ${ }^{6,7}$ afirmam que as jovens assumem comportamento de risco e tornam-se vulneráveis ao praticarem sexo sem a adoção do preservativo por receio de perder o parceiro.

$\mathrm{O}$ aumento de casos de aids entre os jovens tem sido expressivo nos últimos anos. Este fato pode ser associado ao início da vida sexual ativa, em torno de 15-16 anos (detectado pela Pesquisa Nacional de Demografia e Saúde - PNDS 2006), e, também, por ter ocorrido, a partir de 1998, uma inversão na razão de sexo em que incide a aids neste grupo etário. No período de 2000 a 2005, ocorreu uma inversão da razão de sexos do número de casos de aids na faixa etária de 15 a 24 anos $(0,9$ casos em homens para cada caso em mulheres). Em 2012, a taxa de detecção de casos de aids no sexo masculino foi de 15,1/100.000 habitantes e de 8,6 em mulheres. Neste contexto, torna-se preponderante que profissionais e serviços de saúde atentem para as necessidades específicas de saúde e demandas dessa população $0^{4,5}$.

O estudo é relevante, considerando a vulnerabilidade da população jovem às doenças sexualmente transmissíveis, segundo dados do boletim epidemiológico do Ministério da Saúde, devendo ser observadas questões econômicas e sociais que irão determinar a necessidade de atenção mais específica e abrangente a este grupo.

A pesquisa tem o objetivo de identificar as práticas sexuais dos estudantes da graduação em enfermagem, a vulnerabilidade e as práticas adotadas pelos jovens para a prevenção de doenças sexualmente transmissíveis.

\section{REVISÃO DE LITERATURA}

No processo de amadurecimento do ser humano, os indivíduos vivenciam as diferentes etapas da vida e suas características peculiares. A adolescência é uma fase de modificação gradativa da infância para a idade adulta e vem sendo estudada por profissionais que se dedicam ao atendimento de jovens. Considerando que essa fase apresenta limites cronológicos distintos, de acordo com a interpretação dos diversos órgãos oficiais, adotou-se, nesta investigação, a definição do Estatuto da Juventude, instituído pela presidência da república em 2013, que considera jovens as pessoas com idade entre 15 e 29 anos de idade. No referido, documento ficou estabelecido que, para evitar situações de conflito para os jovens na faixa etária de 15 a 18 anos, prevalecerá a a lei $n^{\circ} 8.069$, de 13 de julho de 1990 - Estatuto da Criança e do Adolescente ${ }^{4,8}$.

Entende-se por vulnerabilidade a chance de exposição das pessoas ao adoecimento, sendo amplamente utilizado este conceito no planejamento e elaboração de estratégias de controle das DST e aids. A vulnerabilidade dos diferentes grupos populacionais só pode ser entendida quando se tem a clareza da relevância de um amplo conjunto de aspectos que podem ser agrupados em três esferas, sendo estas: individual, institucional e social ${ }^{9}$. A vulnerabilidade é entendida, portanto, como um estado dinâmico de fragilidades e suscetibilidades em diferentes dimensões, decorrente da interferência de diversos fatores e de construções simbólicas e representacionais ${ }^{10}$.

Neste contexto, sabe-se que a descoberta e a iniciação sexual ocorrem, em geral, na adolescência e que, nesta etapa da vida, a vulnerabilidade está relacionada às características emocionais e psíquicas, pelo desejo de vivenciar coisas novas. Tal fato, associado ao sentimento de imunidade percebido por muitos jovens, os tornam mais suscetíveis em contrair doenças pela prática do sexo inseguro ${ }^{7,11}$. 
O meio socioambiental no qual o adolescente está inserido é outro fator que exerce influência em suas atitudes. Por vezes, assumem comportamento de risco envolvendo, principalmente, o uso de drogas, tabaco, álcool e atividades sexuais. Ao analisar a vulnerabilidade dos jovens às DST, deve-se valorizar a associação entre álcool/drogas e atividade sexual, considerando que, por menor que seja a quantidade de droga utilizada, pode ocasionar alterações na percepção e levar os jovens a tomar decisões errôneas ou realizar práticas sexuais de risco, ficando vulneráveis à gravidez não planejada e às doenças sexualmente transmissíveis ${ }^{12}$.

Pesquisa sobre os conhecimentos, atitudes e práticas na população brasileira (PCAP), divulgada pelo Ministério da Saúde em 2011, revela que 77,6\% dos jovens de 15 a 24 anos têm atividade sexual ativa. Somente $35 \%$ dos jovens investigados, nesta faixa etária, declararam uso regular de preservativo independente da parceria, evidenciando que a Educação em saúde para a prevenção de DST deve ser contínua e abrangente ${ }^{13}$.

Ao mesmo tempo em que as práticas educativas são pouco abordadas, a liberação de costumes e a erotização da mídia vêm estimulando uma iniciação sexual cada vez mais precoce, o que também pode aumentar as chances do jovem contrair DST. Nota-se que, quanto mais jovem é o indivíduo, menor é o nível de informação, tornando-o vulnerável para contrair doenças transmitidas pelo sexo. De acordo com dados do Boletim epidemiológico no período de 1980-2012, há registro da ocorrência de aids em jovens de ambos os sexos nos grupos etários de 15-19 anos e de 20-24 anos, totalizando 12.246 casos e 57.429 respectivamente. As tendências apresentadas, atualmente, pela epidemia evidenciam a heterossexualização, feminização, interiorização e pauperização 4,5 .

A educação em saúde tem, portanto, um papel importante neste cenário, considerando a promoção da saúde para o desenvolvimento da autonomia e a responsabilidade das pessoas e comunidades com a sua saúde. É uma prática social crítica e transformadora, utilizada de forma ampla para a prevenção das DST/ aids. Ainda nos dias de hoje, existe tabu no diálogo entre pais e filhos sobre sexo e sexualidade. Alguns profissionais de saúde, também, demonstram despreparo ao dialogar o tema com adolescentes ${ }^{7,9,14}$.

O enfermeiro tem um papel importante como educador em saúde e realiza uma prática que converge para o contexto sociocultural dos adolescentes. Nas atividades educativas realizadas em um ambiente acolhedor com jovens, são verbalizadas dúvidas, havendo envolvimento, trocas de informações e vivências, resultando na construção do conhecimento coletivo. Essa prática mostra-se eficaz para a aprendizagem de assuntos relacionados à sexualidade e prevenção de DST ${ }^{11,15}$.

\section{Metodologia}

Estudo descritivo, quantitativo realizado com material armazenado no banco de dados da pesquisa Avaliando o conhecimento, as práticas e crenças dos estudantes universitários em relação às doenças sexualmente transmissiveis, coordenado pela Professora Dra. Thelma Spindola. O estudo foi realizado em 2013/2014, em uma universidade pública situada no município do Rio de Janeiro, junto aos estudantes da graduação em enfermagem, maiores de 18 anos, regularmente matriculados na Faculdade de Enfermagem.

Foi aplicado um questionário estruturado, com 50 questões ( 47 fechadas e três abertas), aos estudantes que cursavam do $1^{\circ}$ ao $9^{\circ}$ período acadêmico. Para compor o estudo, utilizou-se amostra do tipo intencional e estratificada, tendo-se selecionado $40 \%$ dos estudantes de cada turma regularmente matriculados na instituição. Inicialmente, foram aplicados 17 instrumentos em cada turma, totalizando 153 questionários. Após a perda de 10 questionários (não devolução) - e com a aplicação dos critérios de exclusão: menores de 18 anos e questionários preenchidos de forma incongruente -, foram excluídos oito instrumentos. Ao final, obteve-se 135 questionários válidos, cujos dados foram armazenados no software Microsoft Excel 2010, e analisados com auxílio da estatística descritiva. Para atender aos objetivos deste estudo, foram utilizados somente questionários de participantes com vida sexual ativa, num total de 89 instrumentos. Do instrumento de coleta de dados aplicado, foram analisadas 16 variáveis relacionadas às características sociodemográficas e ao comportamento sexual dos participantes.

A pesquisa maior foi aprovada pela Comissão de Ética e Pesquisa (COEP) da Universidade do Estado do Rio de Janeiro, com o parecer n ${ }^{\circ}$ 663/2012.

\section{Resultados e Discussão}

\section{Características sociodemográficas}

Os estudantes investigados têm suas características sociodemográficas evidenciadas na Tabela 1.

Os resultados evidenciam que a maioria dos universitários - 76(85,4\%) - era do sexo feminino. Dados do Censo da Educação Superior de 2012, coletados pelo Instituto Nacional de Estudos e Pesquisas Educacionais Anísio Teixeira (INEP), evidenciam que as maiores taxas de escolarização na educação superior são registradas no público feminino ${ }^{16}$. $\mathrm{O}$ aumento da inserção da mulher no ensino superior pode ser relacionado ao crescimento da participação feminina no mercado de trabalho. Segundo o Instituto Brasileiro de Geografia e Estatística (IBGE), a distribuição da 
TABELA 1: Caracterização sociodemográfica dos estudantes universitários. Rio de Janeiro, 2013. (N=89)

\begin{tabular}{lcc}
\hline Aspectos sociodemográficos & $\mathrm{f}$ & $\%$ \\
\hline Sexo & 76 & 85,4 \\
$\quad$ Feminino & 13 & 14,6 \\
$\quad$ Masculino & & \\
Idade & 54 & 60,7 \\
$\quad 18-21$ & 26 & 29,2 \\
$22-25$ & 9 & 10,1 \\
$26-29$ & & \\
Religião & 35 & 39,3 \\
Católica & 19 & 21,3 \\
Evangélica & 7 & 7,9 \\
Espírita & 3 & 3,4 \\
Outra religião & 15 & 16,9 \\
Não possui religião & 10 & 11,2 \\
$\quad$ Não informou & & \\
Estado conjugal & 45 & 50,6 \\
Só namoro & 30 & 33,7 \\
Não tenho namorado & 8 & 9 \\
$\quad$ União estável & 6 & 6,7 \\
União não estável & & \\
\hline
\end{tabular}

população ocupada no país é representada por 63,9\% de mulheres e $61 \%$ de homens na faixa etária de 25 a 49 anos $^{17}$. Nota-se, então, que o quadro da sociedade econômica ativa no país tem se modificado gradualmente, com a participação cada vez mais presente de mulheres no mercado de trabalho, ocupando espaços onde antes havia predomínio do grupo masculino.

Considerando que o grupo investigado era constituído por estudantes de enfermagem, observa-se que este perfil está associado à profissão do enfermeiro, área predominantemente feminina, em função do envolvimento das mulheres com o ato de cuidar e reproduzido até hoje na enfermagem ${ }^{18}$. Quanto à faixa etária, 54(60,7\%) têm idades entre 18 e 21 anos. Estes achados estão em consonância com o último Censo de Educação Superior (2011), sendo evidenciado que a proporção dos jovens de 18 a 24 anos passou de $22,9 \%$ para $47,1 \%$ do final do século passado a 2011 , constatando o aumento da frequência de jovens no ensino superior em todas as regiões do país ${ }^{19}$. Nesse período da juventude, sabe-se que os jovens estão no auge da sua vida sexual ativa, visto que a idade média da iniciação sexual dos brasileiros está em torno dos 15 anos de idade ${ }^{20}$.

O ingresso na universidade é, portanto, um momento de celebração e divertimento com os amigos e, muitas vezes, existe uma maior exposição ao álcool e drogas que, associados às práticas sexuais, podem resultar em uma diminuição do uso da camisinha, considerando que essas substâncias alteram a sensopercepção do indivíduo ${ }^{21}$.

Em relação à religião, 35(39,3\%) participantes declararam ser católicos e 19(21,3\%), evangélicos. A religião assume um papel muito importante para as pessoas porque pode influenciar o modo como os jovens se relacionam no âmbito socioafetivo. O protestantismo e o catolicismo são religiões cristãs e desde os primórdios costumam tratar sexo e sexualidade de forma repressiva, o que pode ocasionar dificuldade para abordar esses assuntos e influenciar a prática sexual dos jovens ${ }^{22}$. Em função das mudanças sociais da atualidade, todavia, acredita-se que mesmo nesses espaços podem estar ocorrendo modificações que favoreçam o diálogo e a reflexão.

Quanto ao estado conjugal dos participantes, 45(50,6\%) declararam que só namoram e 30 (33,7\%), que não têm namorado. $O$ namoro é um relacionamento social e afetivo importante para o desenvolvimento do ser humano. Esse momento, muitas vezes, é associado ao início da vida sexual que, em geral, ocorre na adolescência ${ }^{23}$. É na adolescência que muitos jovens vivenciam o início das atividades sexuais e, nessa ocasião, nem sempre adotam a prática do sexo seguro ficando vulneráveis para contrair uma DST ${ }^{22}$. O estado conjugal declarado é coerente com a faixa etária dos participantes do estudo, considerando que os jovens tendem a buscar relacionamentos mais sérios com idades mais avançadas.

\section{Práticas sexuais}

Os resultados sobre o uso do preservativo segundo o tipo de relacionamento afetivo dos estudantes de enfermagem podem ser visualizados na Tabela 2.

Do total de participantes do estudo, 19(21,36\%) tiveram relação com parceiros casuais, fazendo uso

TABELA 2: Distribuição dos estudantes segundo o uso de preservativo nos últimos 12 meses, e tipo de relacionamento. Rio de Janeiro, 2013.

\begin{tabular}{|c|c|c|c|c|c|c|}
\hline \multirow{3}{*}{ Tipo de relacionamento } & \multicolumn{6}{|c|}{ Uso de preservativo } \\
\hline & \multicolumn{2}{|c|}{ Sim } & \multicolumn{2}{|c|}{ Não } & \multicolumn{2}{|c|}{ Total } \\
\hline & $f$ & $\%$ & $f$ & $\%$ & $f$ & $\%$ \\
\hline \multicolumn{7}{|l|}{ Parceiros casuais } \\
\hline Sim & 18 & 20,23 & 1 & 1,13 & 19 & 21,36 \\
\hline Não & 46 & 51,68 & 24 & 26,96 & 70 & 78,64 \\
\hline Total & 64 & 71,91 & 25 & 28,09 & 89 & 100,00 \\
\hline
\end{tabular}


de preservativo, considerando-se os últimos 12 meses. Sabe-se que, nos dias de hoje, a vida amorosa dos jovens vem sofrendo mudanças nos padrões de comportamento. Antes de se estabelecer um namoro, existe um relacionamento prévio com parceiros casuais, o que pode contribuir para o aumento do número de pessoas com quem o jovem se relaciona sexualmente. Nesses relacionamentos, se a prática do uso do preservativo não ocorrer de maneira contínua, poderá acarretar danos para a saúde desse grupo e se tornar um problema de saúde pública ${ }^{24}$.

Do total de estudantes que tinham vida sexual ativa (89), 46(51,7\%) tiveram relações sexuais com parceiros fixos nos últimos 12 meses e utilizaram preservativos nessas relações; 34(38,2\%) não fizeram uso e $9(10,1 \%)$ não informaram. Embora 46(51,7\%) dos participantes tenham informado a adoção do preservativo, nota-se que os demais jovens - 43(48,3\%) - se mostram resistentes para incorporar essa prática em suas atividades sexuais. Estudo constatou que os jovens fazem uso do preservativo conforme o tipo de relacionamento (parceiro fixo ou casual) e aparência do parceiro, sendo associada ou não à promiscuidade, e empregam o condon como método de contracepção e não para a prevenção de DST ${ }^{25}$.

Embora os participantes da pesquisa utilizem o preservativo com parceiros fixos, ainda é significativo o número de jovens 34(38,2\%) que não o adotam. A não adoção da camisinha nos contatos sexuais pode estar relacionada ao fato de a relação estável envolver confiança no parceiro e esse sentimento ser usado como forma de prevenção. Além disso, nesses relacionamentos, há uma maior utilização da pílula anticoncepcional, demonstrando uma maior preocupação dos jovens em evitar uma gravidez indesejada do que em utilizar preservativos para prevenir doenças sexualmente transmissíveis ${ }^{26}$.

Por se tratar de uma pesquisa cuja maioria dos participantes é do sexo feminino, a não utilização do preservativo pode estar relacionada à dificuldade das mulheres em negociar o uso do preservativo, sob a alegação do medo de perder ou desagradar o parceiro ou passar uma visão de serem experientes ${ }^{27}$. Estudo desenvolvido com mulheres maiores de 18 anos em uma universidade na cidade do Rio de Janeiro identificou que algumas não têm coragem de propor o uso da camisinha, temendo a reação do companheiro, já que o pedido para a utilização do preservativo pode gerar desconfiança em seu parceiro ${ }^{28}$.

A prática do sexo seguro entre os universitários é expressa pelos resultados da Tabela 3 .

Quando se discute a utilização constante do preservativo, pensa-se na subjetividade implicada nesta prática. Os resultados da pesquisa evidenciam que 25(28\%) estudantes não praticam sexo de forma sempre segura e já tiveram mais de um parceiro sexual na vida. Vários fatores podem estar associados a este comportamento e contribuem para que esse grupo seja mais vulnerável às DST e aids. Diversos fatores que levam um indivíduo a não utilizar a camisinha podem ser elencados como: as atitudes negativas relacionadas ao uso do preservativo, alegação da diminuição de sensibilidade e interferência na naturalidade e espontaneidade do ato sexual, o uso de álcool e drogas, o esquecimento de usar e a dificuldade para a negociação de seu uso ${ }^{18,29,30}$. Ao se discutir o emprego do preservativo feminino, sua não utilização tem se apresentado de maneira expressiva. Estudo revelou que as jovens têm dificuldades no uso e manuseio do preservativo feminino, além de seu custo ser elevado quando comparado ao preservativo masculino ${ }^{31}$. Salienta-se, todavia, que os preservativos (masculino e feminino) são distribuídos gratuitamente nas unidades básicas de saúde do município do Rio de Janeiro.

Investigação realizada em Fortaleza, numa instituição pública de ensino superior, em 2010, sinaliza que os jovens são mais vulneráveis às epidemias. Quando se trata de jovens universitários, pode-se associar a vulnerabilidade ao fato de terem ingressado no ensino superior. Por esse motivo, acreditam possuir conhecimento suficiente e não percebem sua exposição às DST. Quando há a associação entre as práticas sexuais e a prevenção de doenças e agravos, essa população mostra-se mais interessada na prevenção de uma gravidez indesejada que evitar uma DST ${ }^{32}$.

Acrescenta-se, também, que existem aspectos subjetivos envolvidos nas práticas sexuais dos indivíduos, assim como a própria sexualidade. A sexualidade está associada a valores e normas sociais que estão atrelados às emoções e instintos. Estes aspectos devem ser

TABELA 3: Práticas sexuais dos jovens universitários. Rio de Janeiro, 2013.

\begin{tabular}{lccccccc}
\hline \multirow{2}{*}{ Práticas sexuais } & \multicolumn{3}{c}{ Já teve mais de um parceiro sexual na vida } \\
& \multicolumn{2}{c}{ Sim } & & \multicolumn{2}{c}{ Não } & \multicolumn{2}{c}{ Total } \\
\cline { 2 - 8 } & f & $\%$ & f & \% & f & $\%$ \\
\hline Pratica sexo de forma segura sempre & & & & & & \\
Sim & 19 & 21,3 & 24 & 27,1 & 43 & 48,4 \\
Não & 25 & 28 & 21 & 23,6 & 46 & 51,6 \\
Total & $\mathbf{4 4}$ & $\mathbf{4 9 , 3}$ & $\mathbf{4 5}$ & $\mathbf{5 0 , 7}$ & $\mathbf{8 9}$ & $\mathbf{1 0 0 , 0 0}$ \\
\hline
\end{tabular}


valorizados, mas, com frequência, não são controlados/ racionalizados no momento do intercurso sexual. $\mathrm{O}$ uso contínuo do preservativo, entretanto, é uma prática que necessita de estímulo para se tornar natural ${ }^{31}$.

No que tange à saúde sexual e reprodutiva dos jovens, o enfermeiro tem um papel importante nas atividades de educação em saúde dos adolescentes, porque estimula o autocuidado, esclarece dúvidas e favorece a integralidade entre os setores da educação e saúde, contribuindo para a redução da vulnerabilidade desse grupo às DST/AIDS ${ }^{33}$. Assim, é importante que o enfermeiro perceba o enfoque da educação em saúde para atuar sob o aspecto de uma educação crítica e transformadora, que contemple as necessidades biopsicossociais em ações individuais e coletivas ${ }^{34}$.

É oportuno salientar que, no exercício de suas funções, o enfermeiro desempenha o papel de educador e continuamente necessita atualizar seus conhecimentos, transformar e/ou ampliar sua prática profissional por meio da educação permanente ${ }^{35}$. Nesse contexto, pode atuar em projetos junto às escolas, participando da educação em saúde de jovens, como o Programa de Saúde nas Escolas (PSE), que auxilia na orientação sexual, prevenção de DST e gravidez de adolescentes da rede pública ${ }^{11}$.

\section{CONClusão}

Os achados do estudo demonstram que os graduandos de enfermagem, semelhante a outros jovens, têm relação com parceiros fixos e casuais. Adotam o preservativo masculino na maioria dos intercursos sexuais. Contudo, existe parte expressiva (mais de um terço) que não pratica sexo de forma sempre segura, ficando vulnerável às DST.

Considerando que o estudo foi realizado com graduandos de enfermagem, com predomínio majoritário de mulheres, fica evidente a relação de gênero na negociação do uso de preservativo com o parceiro e que as mulheres, em geral, sentem dificuldade para negociar esta prática por receio de perder o parceiro. Entre os jovens que tiveram relacionamentos com parceiros casuais, a quinta parte informou ter utilizado o preservativo; entretanto, se considerar que cerca da metade da amostra não pratica sexo de forma sempre segura, este panorama se torna preocupante, especialmente se houver associação de álcool e drogas com a atividade sexual.

A pesquisa atingiu os objetivos à medida que identificou as práticas sexuais de graduandos de enfermagem e a vulnerabilidade desses jovens às doenças sexualmente transmissíveis.

O número de participantes pode ser considerado uma limitação da investigação, todavia os resultados evidenciam a importância de a pesquisa ser replicada em outros espaços, inclusive com estudantes de outras áreas de conhecimento para uma melhor avaliação da temática.

Os achados sinalizam que, embora os participantes sejam estudantes universitários da área da saúde, suas práticas sexuais são semelhantes às de outros jovens e não, necessariamente, associadas ao conhecimento acerca da transmissão das DST. Ratifica-se, então, a realização de ações que estimulem a reflexão dos jovens para uma tomada de decisão consciente em relação à preservação de sua saúde sexual e reprodutiva, com adoção de medidas que favoreçam a prevenção de agravos.

\section{REFERÊNCIAS}

1.Garbin CAS, Lima DP, Dossi AP, Arcieri RM, Rovida TAS. Percepção de adolescentes em relação às doenças sexualmente transmissíveis e métodos contraceptivos. DST J bras Doenças Sex Transm. 2010; 22:60-3.

2.Santos SMJ, Rodrigues JA, Carneiro WS. Doenças Sexualmente Transmissíveis: conhecimento de alunos do ensino médio. DST J bras Doenças Sex Transm. 2009; 21:63-8.

3.Ministério da Saúde (Br). Adolescentes e jovens para a educação entre pares: sexualidades e saúde reprodutiva. Brasília (DF): Ministério da Saúde; 2011. [citado em 10 abr 2015] Disponível em:

http://www.aids.gov.br/sites/default/files/anexos/publicacao/2010/45601/45601/sexualidade_final_17_05_2011_ pdf 28505.pdf.

4.Ministério da Saúde (Br). Secretaria de Atenção em Saúde. Departamento de Ações Programáticas Estratégicas. Diretrizes nacionais para a atenção integral à saúde de adolescentes e jovens na promoção, proteção e recuperação da saúde. Brasília (DF): Ministério da Saúde; 2010. Disponível em: http://bvsms.saude.gov.br/bvs/ publicacoes/diretrizes_nacionais_atencao_saude_adolescentes_jovens_promocao_saude.pdf.

5.Ministério da Saúde (Br). Boletim Epidemiológico HIV/AIDS. Brasília: Ministério da Saúde; 2013. [citado em 15 abr 2015] Disponível em: http://www.aids.gov.br/ sites/default/files/anexos/publicacao/2013/55559/_p_boletim_2013_internet_pdf_p_51315.pdf .

6. Taquette SR, Meirelles ZV. Discriminação racial e as vulnerabilidades às DST/AIDS: um estudo com adolescentes negras. Physis. [online] 2013; 23:129-42. [citado em 15 abr 2015] Disponível em: http://www.

scielo.br/scielo.php?script $=$ sci arttext\&pid $=\mathrm{S} 0103$. $73312013000100008 \& \operatorname{lng}=$ en\& $\bar{n} r m=$ iso.

7.Ministério da Saúde (Br). Saúde e prevenção nas escolas: guia para a formação de profissionais de saúde e de educação. Brasília (Br): Ministério da Saúde; 2010. Disponível em: http://bvsms.saude.gov.br/bvs/publicacoes/saude_prevencao_escolas_guia_formacao_profissionais_educacao\%20.p $\overline{d f}$.

8.Senado Federal (Br). Lei n. 12.852 de 05 de agosto de 2013. Institui o Estatuto da Juventude e dispõe sobre os direitos dos jovens, os princípios e diretrizes das políticas públicas de juventude e o Sistema Nacional de Juven- 
tude - SINAJUVE. [citado em 15 abr 2015] Disponível em: http://www.planalto.gov.br/ccivil_03/_Ato20112014/2013/Lei/L12852.htm.

9.Ayres JRCM, França Júnior I, Calazans GJ, Saletti Filho HC. O conceito de vulnerabilidade e as práticas de saúde: novas perspectivas e desafios. In: Czeresnia D, Freitas CM, organizadores. Promoção da saúde: conceitos, reflexões, tendências. Rio de Janeiro: Fiocruz; 2003. p.17-39.

10.Gomes AMT. A vulnerabilidade como elemento organizador do cuidado de enfermagem no contexto do HIV/Aids - conceitos, processos e representações sociais [tese professor titular]. Rio de Janeiro. Universidade do Estado do Rio de Janeiro; 2011.

11.Fonseca AD, Gomes VLO, Teixeira KC. Percepção de adolescentes sobre uma ação educativa em orientação sexual realizada por acadêmicos (as) de Enfermagem. Esc Anna Nery. 2010; 14:330-7.

12.Oliveira DC, Pontes APM, Gomes AMT, Ribeiro MCM. Conhecimentos e práticas de adolescentes sobre DST/HIV/AIDS. Esc Anna Nery. 2009; 13:833-41.

13.Ministério da Saúde (Br). Secretaria de Vigilância em Saúde. Pesquisa de conhecimentos, atitudes e práticas na população brasileira. Brasília: Ministério da Saúde; 2011. Disponível em: http://bvsms.saude.gov.br/bvs/ publicacoes/pesquisa_conhecimentos_atitudes_praticas_populacao_brasileira.pdf.

14.Gubert FA, Santos ACL, Aragão KA, Pereira DCR, Vieira NFC, Pinheiro PNC. Tecnologias educativas no contexto escolar: estratégia de educação em saúde em escola pública de Fortaleza-CE. Rev. Eletr. Enf. [internet] 2009; 11:165-72. [citado em 15 abr 2015] Disponível em: http://www.fen.ufg.br/revista/v11/n1/v11n1a21.htm. 15.Luna IT, Silva KL, Dias FLA, Freitas MMC, Vieira NFC, Pinheiro PNC. Ações educativas desenvolvidas por enfermeiros brasileiros com adolescentes vulneráveis as DST/AIDS. Cienc. enferm. 2012; 18:43-55.

16.Ministério da Educação $(\mathrm{Br})$. Instituto Nacional de Estudos e Pesquisas Educacionais Anísio Teixeira. Resumo técnico: Censo da educação superior 2012. [citado em 15 abr 2015] Disponível em: http://download. inep.gov.br/download/superior/censo/2012/resumo_tecnico_censo_educacao_superior_2012.pdf.

17.Instituto Brasileiro de Geografia e Estatística. Pesquisa mensal de emprego. 2012; 3-8. [citado em 15 abr 2015] Disponível em: http://www.ibge.gov.br/home/ estatistica/indicadores/trabalhoerendimento/pme_nova/ Mulher_Mercado_Trabalho_Perg_Resp_2012.pdf.

18.Costa LHR, Coelho EAC̄. O cuidado na interface com a sexualidade: uma dimensão interditada durante o processo ensino-aprendizagem de enfermeiras. Caderno Espaço Feminino. 2013; 26:154-77.

19. Travitzki R, Raimundo RLG. Alunos cotistas e atividades extracurriculares: análise do Censo da Educação Superior 2009. R bras est pedag. 2012; 93:77-95.

20.Pereira ALF, Penna LHG, Pires EC, Amado DC. Sexual and birth control health practices among female undergraduates: a descriptive study. Online braz j nurs [internet]. 2014 [cited 2015 May 13]; 13: 25-35. Avail- able from: http://www.objnursing.uff.br/index.php/nursing/ article/view/4286.

21.Custódio G, Trevisol SF, Daisson TJ, Zappelini CE. Comportamento sexual e fatores de risco para a ocorrência de gravidez, DST e HIV em estudantes do município de Ascurra (SC). Arquivos Catarinenses de Medicina. 2009; 38:56-61.

22.Rios LF, Paiva V, Maksud I, Oliveira C, Cruz CMS, Silva CG et al. Cuidados com a carne na socialização sexual dos jovens. Psicol. estud. 2008; 13:673-82.

23.Moreira MRC, Santos JFFQ. Entre a modernidade e a tradição: a iniciação sexual de adolescentes piauienses universitárias. Esc Anna Nery. 2011; 15:558-66.

24. Barreto ACM, Santos RS. A vulnerabilidade da adolescente às doenças sexualmente transmissíveis: contribuições para a prática da enfermagem. Esc. Anna Nery. 2009; 13:809-16.

25.Fontanella BJB, Gomes R. Prevenção da AIDS no período de iniciação sexual: aspectos da dimensão simbólica das condutas de homens jovens. Ciênc saúde coletiva. 2012; 17:3311-22.

26.Alves CA, Brandão ER. Vulnerabilidades no uso de métodos contraceptivos entre adolescentes e jovens: interseções entre políticas públicas e atenção à saúde. Ciênc saúde coletiva. 2009; 14:661-70.

27.Ribeiro KCS, Silva J, Saldanha AAW. Querer é poder? A ausência do uso de preservativo nos relatos de mulheres jovens. DST - J bras Doenças Sex Transm. 2011; 23:84-9.

28.Sampaio J, Santos RC, Callou JLL, Souza BBC. Ele não quer com camisinha e eu quero me prevenir: exposição de adolescentes do sexo feminino às DST/aids no semi-árido nordestino. Saude soc. 2011; 20:171-81. 29.Camargo EAI, Ferrari RAP. Adolescentes: conhecimentos sobre sexualidade antes e após a participação em oficinas de prevenção. Rio de Janeiro: Ciênc. saúde coletiva. 2009; 14:937-46.

30.Cruzeiro ALS, Horta BL, Pinheiro RT, Rocha CLA, Silva RA, Souza LDM. Comportamento sexual de risco: fatores associados ao número de parceiros sexuais e ao uso de preservativo em adolescentes. Ciênc saúde coletiva. 2010; 15:1149-58.

31.Costa JES, Silva CD, Gomes VLO, Fonseca AD, Ferreira DA. Preservativo feminino: dificuldades de adaptação e estratégias para facilitar o uso rotineiro. Rev enferm UERJ. 2014; 22:163-8.

32.Bezerra EO, Chaves ACP, Pereira MLD, Melo FRG. Análise da vulnerabilidade sexual de estudantes universitários ao HIV/aids. Rev Rene. 2012; 13:1121-31.

33.Malta DC, Silva MAI, Mello FCM. Saúde sexual dos adolescentes segundo a Pesquisa Nacional de Saúde dos Escolares. Rev bras epidemiol. 2011; 14:147-56.

34.Sousa LB, Torres CA, Pinheiro PNC, Pinheiro AKB. Práticas de educação em saúde no Brasil: a atuação da enfermagem. Rev enferm UERJ. 2010; 18:55-60.

35.Viana MRP, Moura MEB, Nunes BMVT, Monteiro CFS, Lago EC. Formação do enfermeiro para prevenção do câncer do colo uterino. Rev enferm UERJ. 2013; 21:624-30. 